\title{
Probing the Electronic Structure of Bulk Water at the Molecular Lengthscale with Angle-Resolved Photoelectron Spectroscopy
}

\author{
Samer Gozema, Robert Seidel $\left.\right|^{b, c, y}$, Uwe Hergenhahnd,e,z, Evgeny Lugovoy d,f, \\ Bernd Abeld,f, Bernd Wintere, Anna I. Krylovg,n,", Stephen E. Bradforths, \\ a Department of Chemistry, Georgia State University, Atlanta, GA 30303, USA \\ ${ }^{b}$ Helmholtz-Zentrum Berlin für Materialien und Energie, Albert-Einstein-Straße 15, 12489 Berlin, \\ Germany \\ c Humboldt-Universität zu Berlin, Department of Chemistry, Brook-Taylor-Strasse 2, D-12489 Berlin, \\ Germany, \\ d Leibniz Institute of Surface Engineering (IOM), Chemistry Department, Permoserstr. 15, 04318 \\ Leipzig, Germany \\ e Fritz-Haber-Institut der Max-Planck-Gesellschaft, Faradayweg 4-6, 14195 Berlin, Germany \\ ${ }^{f}$ University of Leipzig, Wilhelm-Ostwald-Institute for Physical and Theoretical Chemistry, \\ Linnéstr. 3, 04318 Leipzig, Germany \\ ${ }^{\mathrm{g}}$ Department of Chemistry, University of Southern California, Los Angeles, CA 90089, USA \\ ${ }^{\mathrm{h}}$ The Hamburg Centre for Ultrafast Imaging, Luruper Chaussee 149, 22671 Hamburg, Germany \\ ${ }^{y}$ Contributed equally \\ ${ }^{2}$ Present Address: Max-Planck-Institut f国ur Plasmaphysik, Wendelsteinstr. 1, 17491 Greifswald, \\ Germany \\ ${ }^{*}$ Corresponding authors
}

\begin{abstract}
Understanding how a solvent affects the electronic properties of solutes is of paramount importance for chemistry. Particularly important are the local solvent structure around solvated molecules and the changes in the shapes and energies of a solute's orbitals induced by the interactions with the solvent. Photoelectron spectroscopy (PES) is a powerful tool for probing electronic structure.(1) The kinetic energies of ejected electrons contain the information about their energy levels within the molecules, whereas the photoelectron angular distribution (PAD) encodes the information about the shape of the orbital from which they originate. $(2,3)$ Although X-ray and ultraviolet PES (XPS $(4)$ and UPS, $(5,6)$ respectively) were originally developed for gas-phase molecules in high or ultrahigh vacuum, (7) the liquid-microjet technique(8) and ambient-pressure instruments(9) have extended PES to the liquid phase, giving rise to an increasing number of XPS and UPS studies of solutions and liquids.(10) Aqueous-phase XPS and UPS experiments can be used to probe how water molecules interact with solutes and with each other. If a solute's intrinsic electronic structure is only weakly perturbed by the solvent, the PAD of the solute would resemble that of the gas-phase species, and the difference between the two could then be attributed to the solvent-induced perturbation of the molecular orbitals of the solute and the elastic scattering of photoelectrons.(11-13) In other words, if the solute maintains its chemical identity, as imprinted in the respective molecular orbitals, then the PAD of solvated species should maintain their gas-phase attributes, even if new features or energetic shifts emerge due to
\end{abstract}


hydrogen bonding or electrostatic interactions. However, what about a neat substance like liquid water? The bulk is likely to have a delocalized valence electronic structure, akin to excitonic bands in molecular solids.(14) Indeed, calculations of water clusters(15-18) have shown delocalization of the valence states over many water molecules, except for valence states at both ends of the band edge. Should one then expect the PAD of bulk water to be entirely different from the PAD of water vapor due to delocalization? Or would water in bulk maintain some semblance of its individual electronic character?

In this work, we attempt to answer these questions by measuring the PAD of the valence states of neat water using high-energy radiation (ranging over several hundreds of electron-volts) and computing the anisotropy parameter $\beta$ from first principles. Although several studies have interrogated these questions by measuring PADs of neat or doped water clusters and microjets, $(11,12,19-23)$ a complete picture is still elusive. The main challenge stems from many competing effects, such as intrinsic changes in the electronic structure, elastic and inelastic scattering, and surface versus bulk sensitivity, that might affect the experimental observables and that are difficult to disentangle theoretically $(24,25)$ (in this context, elastic scattering, for the most part, refers to quasi-elastic scattering, i.e., including electrons inelastically scattered by low-energy phonons but still appearing within the same photoelectron peak). The signatures of these phenomena and the magnitude of the relevant cross sections are system- and energydependent.

It is still not obvious to what extent changes in the PAD of bulk water compared to the gas phase are due to intrinsic changes in the electronic structure of water molecules (i.e., changes in orbital shape due to delocalization, rehybridization, and loss of symmetry) or due to scattering. $(12,13,19-21,23,25,26)$ The work by Thürmer et al.(19) helps to disentangle these two effects by measuring the PAD for ionization of the 1so orbital of water. Because this core orbital should not be significantly perturbed by the other water molecules and should, therefore, retain its shape, any difference between the measured PAD in a microjet and the ideal PAD of $\beta=2$ (at high energies) for an isolated water molecule(27) can be attributed to scattering from neighboring waters. Thürmer et al. have shown(19) that the anisotropy parameter is reduced by about $20 \%$ at photoelectron kinetic energies above $100 \mathrm{eV}$, reaching the asymptotic value of $\beta \sim 1.6$. The reduction of $\beta$ was found to be more pronounced at low energies, in agreement with shorter electron attenuation lengths of slow photoelectrons.(12) We note that the surface sensitivity of the experiments may affect the interpretation of this result in terms of elastic and inelastic mean free paths.(26)

Similar observations were made by Ahmed and coworkers, $(28,29)$ who studied PADs from the $1 s$ core orbitals in a variety of nanoparticles. They found that while $\beta$ is substantially reduced at low kinetic energies, the elastic mean free path increases considerably at high electron energies, resulting in $\beta \approx 2$ expected for ionization of 1 s orbitals in the absence of scattering.

The results of these earlier studies $(12,19)$ provide an important stepping stone for the present work: it is reasonable to expect that the extent of scattering should be the same for the ionization of the valence bands of water, with a similar electron kinetic energy as for the 1so core orbital, so any further reduction in the magnitude of $\beta$ for the valence orbitals beyond that seen for 1 so can be ascribed to the changes of the molecular orbitals upon solvation. 
Recently, Signorell and coworkers investigated valence PADs in water clusters and microjets, $(11,21,23)$ with the goal of disentangling different phenomena affecting PADs and determining the convergence of the anisotropy to the bulk limit. They observed that, at low energy, $\beta$ rapidly decreases with the cluster size and concluded that essentially bulk-like electronic structure is attained in clusters of 5-6 water molecules. Consistent with their cluster results and modeling of scattering, their extrapolation to bulk yielded a substantially reduced $\beta .(21,23)$ The crucial difference between the present paper and the work of Signorell and coworkers is the energy range: the latter study employed low-energy radiation, giving rise to photoelectrons much below $100 \mathrm{eV}$. As discussed in this Letter, this detail leads to strikingly different results.

Figure $1 \mathrm{~B}$ shows total valence PES of neat water using a microjet at a photon energy of $265 \mathrm{eV}$. Figure S1 in the Supporting Information shows an example of the spectrum as a function of $\theta$, which represents the angle between the polarization axis of the incoming light and the orientation of the electron analyzer. Experimental details of our setups can be found in the Supporting Information.

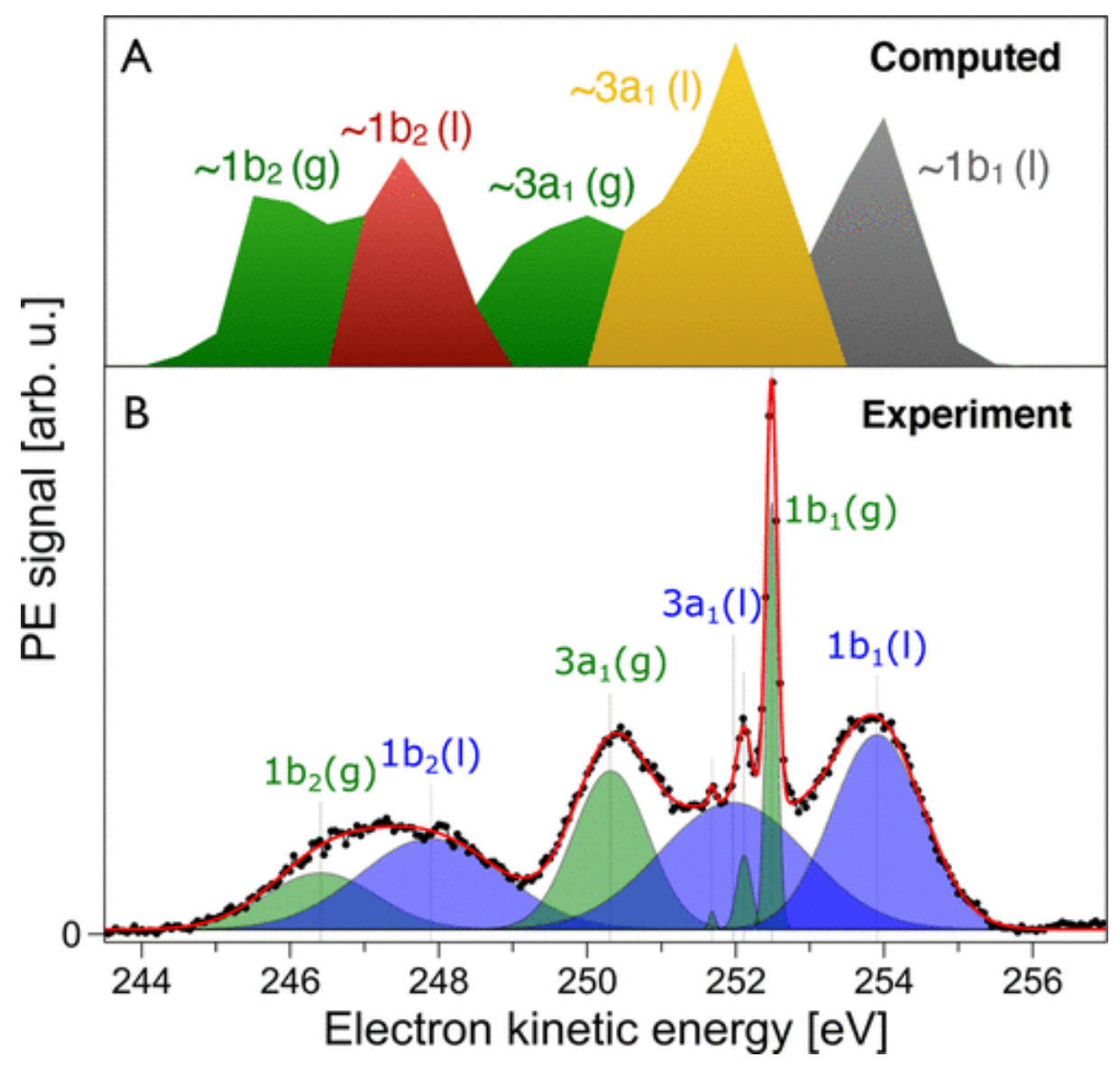

Figure 1. Photoelectron spectrum of liquid water. Panel A: Theoretical PES computed with EOM-IP-CCSD using pentamers extracted from equilibrium MD simulations of liquid water. The $1 b_{1}$ band (gray) is defined as any state in the 9.5-12.5 eV ionization energy range, the $3 a_{1}$ band (yellow) is defined as any state in the $12.5-15 \mathrm{eV}$ range, and the $1 \mathrm{~b}_{2}$ band (red) is defined as any state in the 16.5-18.5 eV range. Panel B: Deconvolution fit of the experimental PES (symbols) obtained at $h v=265 \mathrm{eV}$ and $\theta=0$. The fit gives peaks corresponding to ionization from the $1 b_{1}, 3 a_{1}$, and $1 b_{2}$ orbitals of water for gas phase (green peaks) and liquid (blue peaks). 
The spectral bands (Figure 1 and Figures S1 and S2 in the Supporting Information) can be assigned to the ionization from the three highest-energy molecular orbitals of isolated (gasphase) water molecules and of liquid water. The analysis of the PADs confirms that, as in the core-ionization study,(19) the standard equation (eq S1 in the Supporting Information) derived for randomly oriented molecules is valid and that the PAD is determined entirely by the dipole anisotropy parameter $\beta$. $\beta$ values associated with each peak are extracted from fitting the gasphase features in our spectra.

$\beta$ values corresponding to the gas-phase peaks are shown in Figure 2 along with the wealth of literature values that correspond to electron kinetic energies up to 100 eV. $(30,31)$ Our HHG result at low photon energy, shown by a green dot in Figure 2, agrees well with the previous measurements; these HHG $\beta$ values refine the earlier published values by Faubel et al.,(32) which were measured in the same lab but lacked a complete set of polarization angles and a proper error analysis. The experimental data from this work are summarized in Tables S1-S3 in the Supporting Information. 

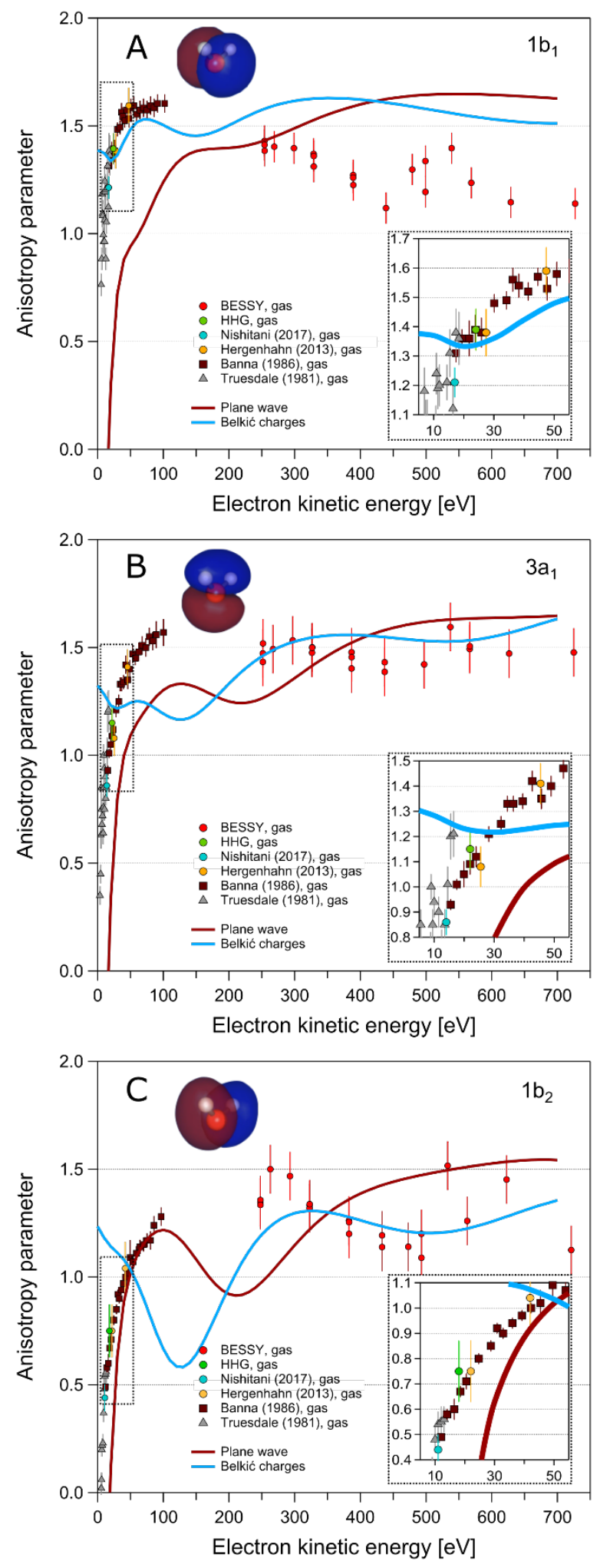

Figure 2. Experimental (symbols) and computed (solid lines) $\beta$ values for ionization of the $1 b_{1}$ (panel A), $3 a_{1}$ (panel B), and $1 b_{2}$ (panel C) orbitals of vapor-phase water over a range of $700 \mathrm{eV}$. Experimental data are from Truesdale et al.(30) (gray triangles), Banna et al.(31) (brown squares), Nishitani et al.(22) (cyan circles), Hergenhahn and coworkers(20) (yellow circles), and this work (HHG data in green; BESSY data in red). Computed anisotropies are shown as solid lines using a plane wave (red) and Coulomb wave with Belkićs charges (blue) for the description of the photoelectron. The corresponding EOM-IP-CCSD Dyson orbitals are shown for each transition. 
The PAD of the valence photoelectrons in gas-phase water $(30,31)$ can be explained by the shapes of the respective molecular orbitals. The $1 b_{2}, 3 a_{1}$, and $1 b_{1}$ orbitals of water have $p$-like character: the $1 b_{1}$ orbital is a pure $p$-like lone pair orbital whereas $1 b_{2}$ and $3 a_{1}$, which have $\sigma_{O H}$ character, are slightly distorted by the hybridization and contributions of the $1 s_{\mathrm{H}}$ orbitals. Consequently, for all 3 transitions we expect predominantly an $s$-wave $(\beta=0)$ at low electron kinetic energies and interfering $s$ - and $d$-waves $(\beta>1)$ at higher energies. This is exactly what is observed here and in the previous experiments, $(30,31)$ as well as in the calculations using correlated Dyson orbitals and simple photoelectron description (similar trends were reported in an earlier study using density functional theory(27)). Thus, PADs of gas-phase water clearly show the atomic provenance of the molecular orbitals, while reporting on the variable extent of their hybridization in a molecular environment (e.g., out of the 3 orbitals, the shape of the $1 b_{2}$ orbital is most deformed, leading to the largest deviations of $\beta$ from those expected from a pure $p$-orbital).

The theoretical $\beta$ values in Figure 2 were computed using correlated Dyson orbitals, which encode the information about electronic structure before and after ionization, with the ejected photoelectron wave function treated either as a free particle (plane wave) or as a particle perturbed by the Coulomb potential of the water cation (Coulomb wave). The theoretical framework is described in detail in refs (33and34) and in the Supporting Information. Although the outgoing electron experiences asymptotically $a+1$ charge, the Coulomb potential in the vicinity of the target is different due to the screening of the polyatomic nuclear potential by the remaining electrons. The effect of the screening can be accounted for by using effective charges $(33,35,36)$ and a multicenter expansion. $(35,37-39)$ Figure 2 shows the results computed with a plane-wave approach $(Z=0)$ and with physically motivated effective charges, $Z_{\text {eff }}$ computed by Belkić's rule,(36) based on the generalization of the Rydberg formula for the energy levels of a hydrogen-like atom (Figure S9 in the Supporting Information shows the results with other values of $\left.Z_{\text {eff }}\right)$.

In the low-energy regime, where slow outgoing electrons interact with the perturbing molecular potential for a longer time, such a simple treatment of photoelectrons leads to large errors and large differences between the plane-wave and Coulomb-wave treatments. However, at large energies, fast photoelectrons are less sensitive to the shape of the perturbing cationic potential, such that one can anticipate smaller errors due to the approximate treatment of the free-electron state. Indeed, the difference between the two computational models is reduced above kinetic energies of $\sim 100 \mathrm{eV}$. While neither the plane- nor the Coulomb-wave calculations quantitatively reproduce the experimental data, the plane-wave model reproduces the sharp rise in $\beta$ at low kinetic energies, while the Coulomb wave with Belkićs charges gives the best agreement at high kinetic energies (mean absolute deviation in $\beta$ of $0.28,0.07$, and 0.10 for $1 b_{1}, 3 a_{1}$, and $1 b_{2}$, respectively). This indicates that the simple single-center expansion model employed here captures the variations of the angular distribution of the different ionized states of water using the corresponding Dyson orbitals, which describe deformation of atomic orbitals within a molecular environment. Because the errors appear to be systematic (theory, on average, consistently overestimates $\beta$ values for all 3 bands), we anticipate that this trend will propagate to the bulk calculations and that the magnitude of the reduction in $\beta$ could be captured, despite the discrepancy in absolute values. We employ Coulomb waves with Belkićs charges ( $Z_{\text {eff }}=1.93,2.06$, and 2.30 for $1 b_{1}, 3 a_{1}$, and $1 b_{2}$, respectively) for the rest of the calculations in this work. 
How should we modify this simple picture for a water dimer, larger clusters, and eventually bulk? The measurements by Signorell and coworkers $(21,23)$ on water valence bands suggest that angular anisotropies in the bulk are quickly washed out, giving rise to strongly reduced $\beta$, whereas our PADs (presented below) show a persistent anisotropy consistent with a $p$-like character of the respective Dyson orbitals. To understand these seemingly contradictory findings for bulk water PADs, we start by discussing the theoretical framework for treating photoionization in molecular aggregates (dimers, trimers, etc.). Conceptually, should we think about photoionization of the dimer as a sum of independent waves coming from each monomer or a single wave corresponding to ionization of an entangled dimer state? Do the coherences between the waves coming from the monomers survive, or can we treat the dimer photoionization as a sum of two incoherent waves?

The difference between the two frameworks is illustrated in Figure 3 . Figure $3 \mathrm{~A}$ shows $\beta$ computed for the state derived from the $1 b_{1}$ monomer states of two noninteracting water molecules. For symmetry-identical monomers, the dimer states are two degenerate states corresponding to in-phase and out-of-phase linear combinations of monomer states. The PAD computed using each of these delocalized states and a single-center expansion placed in the centroid of the Dyson orbital (i.e., in between the two fragments) gives $\beta \sim-0.5$ (red curves in Figure 3A). However, for degenerate states, any linear combination can be used, so instead of the delocalized states one can consider two localized Dyson orbitals as equally legitimate solution. The respective PADs derived from the localized states (black curve in Figure $3 A$ ) are identical to those of the monomer (Figure $2 A$ ), giving rise to $\beta \sim 1.5$. For the two infinitely separated waters, the localized description must be the correct one, but at short distances, one may expect the delocalized picture to be more appropriate. The key question is then which model applies better to the photoionization of bulk water. Are molecules in the liquid far enough apart to justify a monomer-based treatment of photoionization, or should one use delocalized orbitals? 

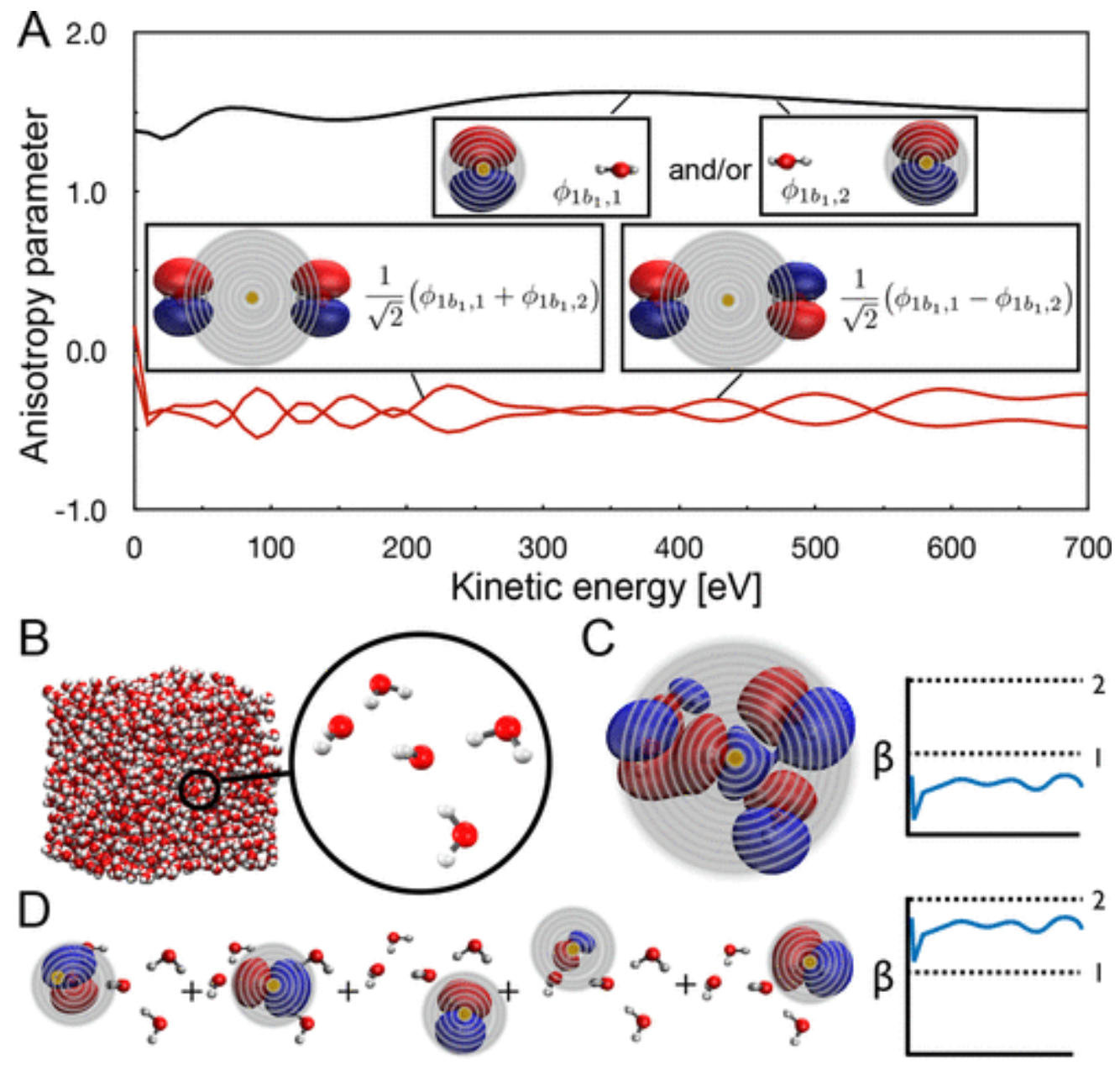

Figure 3. Panel A: $\beta$ values computed for two noninteracting water molecules using localized monomer states (black) and delocalized dimer states (red). Yellow dots indicate the center of the expansion for the free electron state, which are placed at the centroids of the corresponding Dyson orbitals. Panels B-D extend these two frameworks to a water pentamer. One-hundred pentamer geometries were extracted from a molecular dynamics simulation of liquid water (panel B). For each pentamer, $\beta$ values were computed using a single-center expansion (shown by a yellow dot) placed at the centroid of the delocalized Dyson orbital (panel C) or a multicenter expansion (yellow dots mark the centroids of the localized Dyson orbitals) in which the total PAD is given by the sum of the PADs from each water (panel D). The delocalized approach (panel C) yields more isotropic PADs, while the localized approach (panel D) yields more anisotropic PADs consistent with the $p$-like character of the Dyson orbitals.

This exact question has been posed and discussed by Sanov and coworkers(40) using photodetachment from dissociating $\mathrm{I}_{2}{ }^{-}$, which the authors described as a "molecular interferometer". They have shown that at large I-I distances, the PAD corresponds to the atomic-like signal (detachment from a $p$-orbital), whereas at short distances the PAD reflects a delocalized $\pi^{*}$ molecular orbital (which has the same symmetry as a $d$-orbital placed at the bond midpoint). The latter situation can also be described as the interference of the two coherent waves coming from each atom. The transition between the two regimes depends on the photoelectron energy, which determines de Broglie's wavelength $(\lambda)$ of the photoelectron: if the distance between the two centers $(R)$ is larger than $\lambda$, then one can treat the photodetachment as two independent noninterfering waves, whereas for $\lambda \sim R$, the two waves interfere, and the photodetachment signal reveals an entangled state. One can arrive to this 
intuitive result formally by analyzing the effect of coherences between the two centers using the multicenter treatment of photoionization $(35,37)$ (the derivation is given in the Supporting Information). The mathematical reason that the coherences are diminished at high energy is rooted in the oscillatory behavior of the phase between the two waves: the frequency of the oscillations equals $2 E V R 2 \pi$

, where $E$ is the energy of the photoelectrons. When this frequency is large, the coherences are washed out by small variations of $R$ (due to molecular vibrations or thermal motions in the bulk). However, for small frequencies (when de Broglie's wavelength is shorter than $R$ ), the coherences between the waves coming off the two centers cannot be ignored. To reconcile this multicenter treatment with a single-center delocalized picture, it is instructive to think about the coherences in the energy domain. At short distances, the energy gap between the two delocalized states of the dimer is large, and one can ignore the coherences between the two waves corresponding to photoionization of each state; this justifies the use of a single-center delocalized treatment. However, at large distances, the two delocalized states are degenerate and cannot be treated independently (in other words, the coherences between them cannot be ignored). This is why the delocalized single-center expansion is physically incorrect when the energy gap between the delocalized states is small relative to the bandwidth of the ionizing pulse. Put differently, if the coherences between the photoelectrons produced from the two states are incorporated into the calculations, the correct behavior would be obtained, following the single-center result at low energies and the multicenter result at high energies. Therefore, the magnitude of coherences depends on the representation. In the spatial domain (localized multicenter representation), coherences can be ignored when the two centers are far apart relative to de Broglie's wavelength of the photoelectron. In the energy domain (delocalized single-center expansion), coherences can be ignored when the two centers are close and the energy gap between the delocalized states is large.

Below, we discuss the implications of these two distinct regimes in photoionization of bulk water. To simulate the photoionization of bulk, we consider a model system, a water pentamer cut out from an equilibrium molecular dynamics simulation of liquid water (Figure 3B). To account for thermal motions, we average the results over 100 random snapshots. To model the photoelectron spectra, we compute the EOM-IP-CCSD ionization energies and Dyson orbitals for all valence states for each of the 100 pentamer structures. The computed valence photoelectron spectrum is shown in Figure 1A. For comparison, Figure $1 \mathrm{~B}$ shows a measured valence photoelectron spectrum. The features in the experimental spectrum are broken into the contributions from the liquid and gas-phase water molecules and clearly show distinct bands corresponding to the 3 valence molecular orbitals of water in each phase. The computed spectrum also shows the features of both liquid- and gas-phase peaks. Three of the peaks have similar energies and shapes as the experimental spectra of bulk water and are shown in gray $\left(1 b_{1}\right)$, yellow $\left(3 a_{1}\right)$, and red $\left(1 b_{2}\right)$. Additional peaks (marked by green) in the computed spectrum have energies and shapes that are more similar to the experimental spectra for gas-phase water. These peaks originate from the orbitals localized on surface molecules, which resemble gas-phase water more than bulk water. Overall, Figure $1 \mathrm{~A}$ illustrates inhomogeneous broadening of $2-4 \mathrm{eV}$ of the individual bands, leading to their partial overlap.

To simulate the PAD, we computed PADs for each pentamer structure using two different approaches illustrated in Figure 3. In the first approach, we used delocalized Dyson orbitals and a single-center plane-wave expansion (Figure $3 \mathrm{C}$ ); in this approach, orbital deformation and 
interference between the immediate neighbors are accounted for, but the coherences between the nearly degenerate electronic states are ignored. In the second approach, we split the Dyson orbital into the fragments' contributions, each localized on a single water molecule (hereafter referred to as the multicenter or localized model). We compute the differential cross section for each of the fragment orbitals in the pentamer using a Coulomb-wave expansion with Belkić's charges placed on the respective fragment, and then sum up the contributions of the five water molecules to the total PAD (Figure 3D); in this approach, orbital deformation is included, but the interference between neighboring water molecules is ignored. The scattering from nearby waters (i.e., the perturbation of the outgoing electron by the potential of the cluster) is neglected in both models.

Figure 4 shows the computed and experimental $\beta$ values as a function of kinetic energy for the liquid peaks in the photoelectron spectrum. Also shown are the HHG data from this work (35.6 $\mathrm{eV}$ photons) and the data by Nishitani et al.(22) (29.5 eV photons) for the 3 valence states of liquid water. The experimental $\beta$ values are derived by fitting the peak areas for the individual water valence bands as a function of the angle between the electron analyzer and the polarization direction. The data shown include 4 independent runs with 12 different photon energies from the synchrotron and a lower photon energy data set recorded with HHG radiation. The error bars reflect the fluctuations of the liquid jet position, the fitting errors, and the deviations from the ideal polarization provided by the UE52-SGM-1 beamline. Due to a large peak-overlap and fit constraints, the error bars for the $3 \mathrm{a}_{1}$ and $1 \mathrm{~b}_{2}$ orbitals are larger than that for the $1 b_{1}$ orbital. Details about the data acquisition, data analysis, fitting routine, and the error bar determination are given in the Supporting Information. 

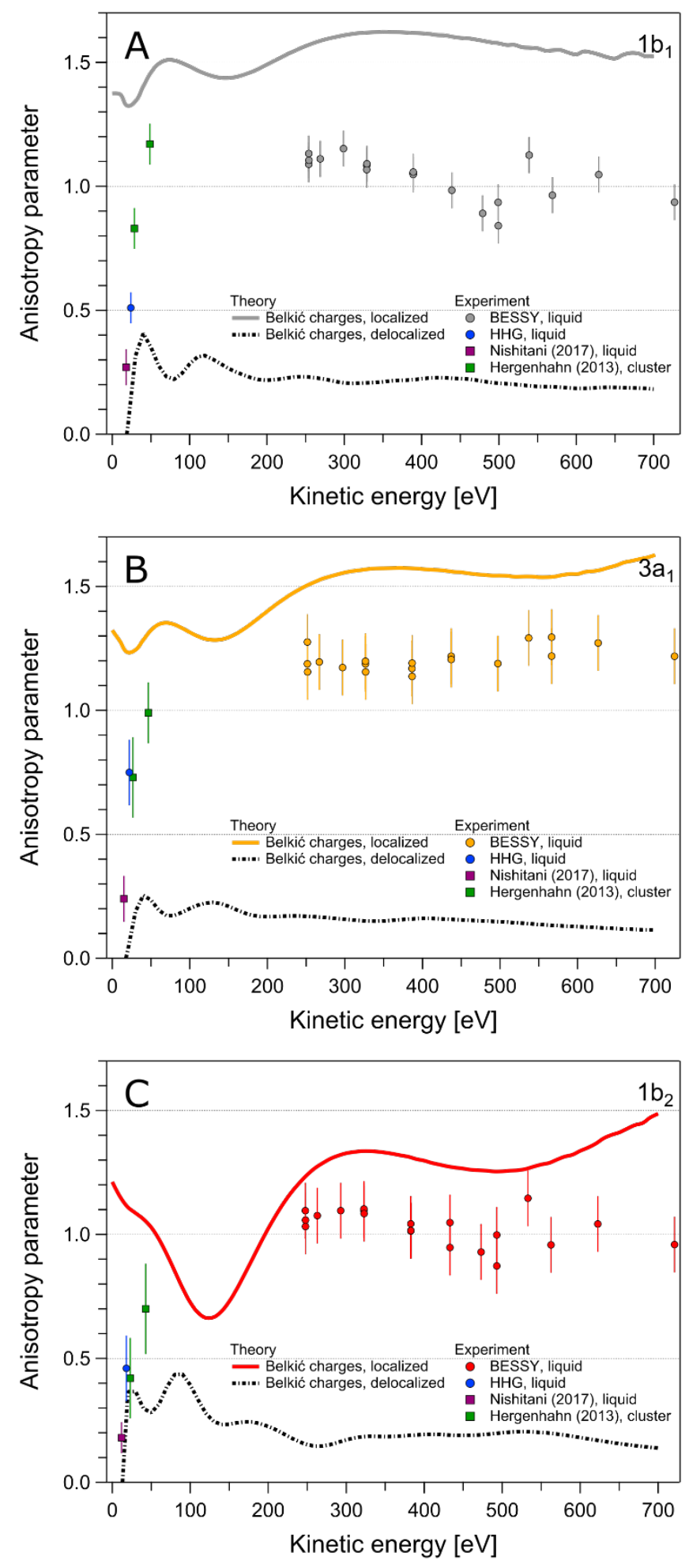

Figure 4. Experimental (symbols) and computed (lines) $\beta$ values as a function of kinetic energy for valence ionization of liquid water. Gray, yellow, and red denote the values for the $1 b_{1}, 3 a_{1}$, and $1 b_{2}$ bands. Theoretical $\beta$ values are assigned based on the energy of the binding energies (see Figure 1 and associate caption) and are computed using both the delocalized model with plane waves (dashed lines) and the localized model using physically motivated Belkić's charges (solid lines), as shown in Figures $3 \mathrm{C}$ and $\underline{3} \mathrm{D}$, respectively. Experimental data are from Nishitani et al.(22) (liquid microjet, magenta squares), Hergenhahn and coworkers $\underline{(20)}$ (water cluster, green squares), and this work (HHG indicated by blue circles, BESSY data with circles, color coded depending on the orbital). 
The experimental $\beta$ values reveal considerable similarity between the bulk (Figure 4 ) and gas phase (Figure 2). At high kinetic energies (i.e., above $250 \mathrm{eV}$ ), $\beta$ for bulk ionization ranges around $1-1.25$, to be compared with the 1.25-1.5 range for the gas-phase ionization. In both cases, the largest $\beta$ values are observed for the $3 a_{1}$ band.

The theoretical $\beta$ values computed using a single delocalized Dyson orbital (dashed line) are strikingly different from those computed by using fragment orbitals and the multicenter expansion (solid lines). The multicenter approach gives $\beta$ values that are in much better agreement with the experiment for electron kinetic energies above $40 \mathrm{eV}$, while the singlecenter expansion model is qualitatively incorrect. $\beta$ values computed using the multicenter model systematically overestimate, by at least $\sim 20 \%$, the experimental bulk values. We attribute this discrepancy to scattering effects, which are not included in the theoretical treatment.

To understand the implications of the results shown in Figure 4, let us revisit the analysis of the two limiting regimes of the dimer photoionization (Figure $3 \mathrm{~A}$ ). A qualitatively incorrect $\beta$ obtained for delocalized Dyson orbitals indicates that in the present experimental regime, bulk water ionization can be described as incoherent superposition of the photoionization of individual water molecules rather than photoionization of delocalized entangled states. We can understand this in the context of Sanov's molecular interferometer, $(40)$ where the short-time evolution of time-resolved photoelectron images from dissociating $\mathrm{I}_{2}{ }^{-}$are discussed in terms of the interference between the two waves coming from the individual atoms. This interference can be described as ionization of a single entangled state spanning both iodine atoms at a short $\mathrm{I}_{2}{ }^{-}$bond length. However, at longer times (i.e., at large separations between the atoms), the variation in $\beta$ ceases and it approaches the asymptotic value corresponding to the PAD of $\mathrm{I}^{-}$. The distance at which the atoms become independent and the coherences die off is related to the energy of the ionizing radiation, which in turn determines the energy of the photoelectrons. In Sanov's study the distance at which the iodine atoms are no longer coherently ionized (or, said another way, at which the photoelectrons no longer interfere) was found to be $35 \AA$, which was in excellent agreement with de Broglie's wavelength of the ejected photoelectrons $(0.12$ $\mathrm{eV}$ in their study).

In bulk water, the typical distance between the nearby molecules is on the order of $3 \AA$. This corresponds to an electron kinetic energy of $\sim 17 \mathrm{eV}$ (see Figure S7). At this energy or lower, the photoionization would likely probe a coherent delocalized state of water (or, using alternative language, reflect the interference between the photoelectrons ejected from the individual fragments). This is consistent with the data reported at low kinetic energies by Signorell and coworkers $(23)$ that show a significant reduction in the $\beta$ of valence bands from large water clusters. Therefore, at low energies, the single-center expansion of the photoelectron wave function is justified. In contrast, at high energies (e.g., above $150 \mathrm{eV}$ where the photoelectron wavelength is $1 \AA$ ), it is more appropriate to describe photoionization using localized orbitals. The observed persistent anisotropy of photoelectrons confirms that water's valence orbitals retain their identity, despite being slightly distorted (polarized and hybridized) by their environment. This implies that PES and photoelectron imaging can probe the local electronic structure of a molecule even in bulk, as long as the ejected photoelectron has a de Broglie wavelength smaller than the proximity of nearby solvent molecules. 
To quantitatively analyze the reduction of anisotropies in bulk relative to the gas phase, we consider the reduced $\beta$ defined as

Bred $\equiv 1-B, B_{g}$

(1)

where $\beta_{\text {I }}$ and $\beta_{\mathrm{g}}$ denote liquid- and gas-phase anisotropies. If the anisotropy in the liquid phase is the same as in the gas phase, then $\beta_{\text {red }}=0$. Conversely, $\beta_{\text {red }} \approx 1$ signifies a complete loss of anisotropy in liquid. The water core-ionization study $(19)$ showed that $\beta_{\text {red }}$ decreases as a function of photoelectron kinetic energy and at high kinetic energies (above $200 \mathrm{eV}$ ) $\beta_{\text {red }} \approx 0.2$. Ahmed and coworkers $(28)$ observed a similar trend in $\beta$ for the $1 s c$ ionization in squalene nanoparticles (at their highest kinetic energy of $\sim 40 \mathrm{eV}, \beta \approx 1$, corresponding to $\beta_{\text {red }} \approx 0.5$ ).

The reduction of the anisotropy for core ionization at high energies has been attributed entirely to elastic scattering, because the shape of the compact $1 s_{0}$ orbital is not perturbed by the interactions with neighboring waters.(19) Our calculations of the anisotropy for 1 so ionization of water pentamers (Figure S13) yield $\beta=2$ at electron kinetic energies above $20 \mathrm{eV}$, thus providing computational support to this conjecture. Our model does not capture the slow rise at low energies (observed both in the gas phase and in bulk), which can be attributed to scattering of photoelectrons from the hydrogens. As evident from the experimental result(19) and from the calculations by Decleva and coworkers for the isolated water molecule,(27) the intramolecular scattering becomes irrelevant at $\sim 100 \mathrm{eV}$, considerably below the estimate based on the de Broglie equation (164 eV, corresponding to the $\mathrm{OH}$ distance).

Figure 5 shows the experimental and theoretical $\beta_{\text {red }}$ values for core and valence ionization. As in the core-ionization study, (19) the experimental $\beta$ values for liquid water show a systematic reduction for valence photoionization. Within the experimental error bars, $\beta_{\text {red }} \approx 0.2$ at high energies for both the 1 so and valence orbitals (compare the $\beta$ values for the data from BESSY in this work with the light-blue curve derived from Thürmer et al.). This is surprising because one might expect that the valence orbitals would undergo a further reduction in $\beta$ upon solvation compared to the core 1 so orbital due to delocalization or distortion of the orbital shape. However, the theoretical $\beta_{\text {red }}$ from the localized model is zero for all three bands, meaning that the interactions with bulk have a rather small effect on the shapes of orbitals of the individual waters, despite significant energetic perturbation, as manifested by the large shifts in the binding energies (by about $1.5 \mathrm{eV}$, with small variations depending on the band(41)) and by inhomogeneous broadening of all three valence bands due to solvation (Figure 1). Thus, despite the participation of the valence orbitals in hydrogen bonding, their shapes remain similar to the shapes of the orbitals in isolated water molecules, and the reduction of $\beta$ at high kinetic energies can be attributed solely to scattering, as in the case of core photoionization. 

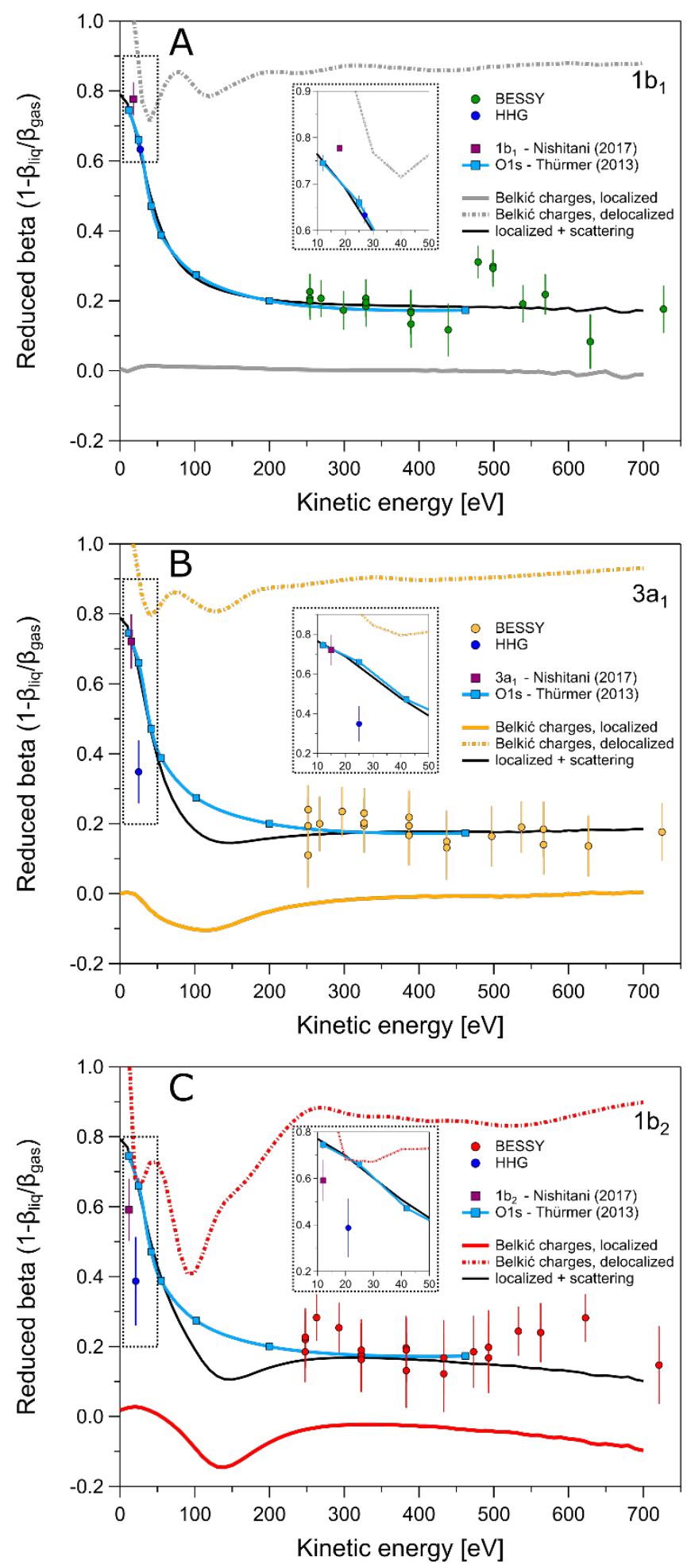

Figure 5. Experimental (symbols) and theoretical (lines) reduced $\beta$ values for the $1 b_{1}$ (panel $A$ ), $3 a_{1}$ (panel B), and $1 b_{2}$ (panel $C$ ) bands of water. The blue squares and solid blue line correspond to the reduction in anisotropy due to scattering extracted from the water 1so core-ionization study.(19) The insets show HHG data points (35.6 eV photons, blue circles) and data by Nishitani et al.(22) (29.5 eV photons, magenta squares). Theoretical data are shown using both the localized model (solid lines) and delocalized model (dashed lines) for each state. The black lines are the theoretical $\beta$ values from the multicenter model corrected for the reduction of $\beta$ due to scattering extracted from the water $1 s_{0}$ experimental data.(19) 
As expected, the results for the single-center expansion show almost a complete loss of anisotropy ( $\beta_{\text {red }} \approx 0.8-0.9$ at high energies). While this treatment is inappropriate for the high kinetic energy regime, at lower energies it captures interference effects ignored in the multicenter (localized) treatment. We note that for the lower-energy data points from Nishitani et al.(22) and for the HHG data points, the delocalized model is in very good agreement with the experiment for all three bands.

To include the effect of scattering in the model, we use the 1 so data from Thürmer et al. Attributing all loss of anisotropy in the 1 so ionization to scattering, we use the ratio of $\beta\left(1 s_{0}\right)_{g} / \beta\left(1 s_{0}\right)$, (fitted using a sigmoidal function) to scale the theoretical values from the localized model. The resulting curves (shown as black solid lines in Figure 5) agree very well with the experimental data, further confirming our interpretation.

In conclusion, we presented a combined experimental and theoretical study of valence photoionization of liquid water using X-ray radiation. The results show that using sufficiently high photon energy enables probing the electronic structure of individual water molecules within the bulk. The decrease in anisotropy values in bulk relative to gas-phase water, which can be attributed to the deformation of valence orbitals due to rehybridization, loss of symmetry, and scattering, is remarkably small. The high-level ab initio calculations show that, despite the large energetic perturbation, solvation and hydrogen bonding interactions have a negligible effect on the shape of molecular orbitals. Thus, the reduction of anisotropies can be attributed mostly to scattering. The comparison with the core-level study further confirms this conclusion.

These results provide an important contribution toward developing a comprehensive picture of photoionization in the condensed phase and highlight the need for developing a unifying theoretical framework seamlessly connecting the low- and high-energy regimes. On the experimental side, more data at intermediate photoelectron energies will help to clarify whether effects of hydrogen bonding and electronic delocalization would be more pronounced at lower energies. To better assess possible effects due to the surface waters and backscattering of the photoelectrons emanating from gas-phase molecules, experiments with different geometries of the jet (e.g., flat jets) are desirable. Such experiments are currently under way.

\section{Acknowledgements}

The authors thank the HZB staff for support during the beamtimes at BESSY II and Johann Nilsson for help with acquisition of the HHG data. U.H., B.A., and B.W. acknowledge funding by the Deutsche Forschungsgemeinschaft (DFG) in the framework of D-A-CH (project SDynG-LI). R.S. acknowledges an Emmy Noether Young Investigator stipend through the DFG (project SE 2253/3-1). A.I.K. has been supported by the Army Research Office through grant W911NF-161-0232. A.I.K. is also a grateful recipient of the 2019 Simons Fellowship in Theoretical Physics and of the Mildred Dresselhaus Guest professorship from the Center for Ultrafast Imaging in Hamburg, which supported her sabbatical stay in Germany. S.E.B. is supported by the U.S. National Science Foundation (CHE-1301465). S.G. acknowledges the donors of the American Chemical Society Petroleum Research Fund for support through PRF 60837-DNI6. 


\section{Notes}

The authors declare the following competing financial interest(s): A.I.K. is the President and a part-owner of QChem,Inc.

\section{References}

(1) Simons, J. In Photoionization and Photodetachment; Ng, C. Y., Ed., Vol. 10, Part II of Advanced Series in Physical Chemistry; World Scientific Publishing Co.: Singapore, 2000.

(2) Sanov, A.; Mabbs, R. Photoelectron imaging of negative ions. Int. Rev. Phys. Chem. 2008, 27, 53-85.

(3) Reid, K. L. Photoelectron angular distributions. Annu. Rev. Phys. Chem. 2003, 54, 397-424.

(4) Nordling, C.; Sokolowski, E.; Siegbahn, K. Precision method for obtaining absolute values of atomic binding energies. Phys. Rev. 1957, 105, 1676.

(5) Vilesov, F. I.; Kurbatov, B. L.; Terenin, A. N. Electron distribution over energies in photoionization of aromatic amines in gaseous phase. Sov. Phys. JETP 1961, 6, 490.

(6) Turner, D. W.; Jobory, M. I. Determination of ionization potentials by photoelectron energy measurement. J. Chem. Phys. 1962, 37, 3007.

(7) Fransson, T.; Harada, Y.; Kosugi, N.; Besley, N. A.; Winter, B.; Rehr, J. J.; Pettersson, L. G. M.; Nilsson, A. X-ray and electron spectroscopy of water. Chem. Rev. 2016, 116, 7551-7569.

(8) Winter, B.; Faubel, M. Photoemission from liquid aqueous solutions. Chem. Rev. 2006, 106, $1176-1211$.

(9) Salmeron, M.; Schlögl, R. Ambient pressure photoelectron spectroscopy: A new tool for surface science and nanotechnology. Surf. Sci. Rep. 2008, 63, 169-199.

(10) Seidel, R.; Winter, B.; Bradforth, S. E. Valence electronic structure of aqueous solutions: Insights from photoelectron spectroscopy. Annu. Rev. Phys. Chem. 2016, 67, 283-305.

(11) Luckhaus, D.; Yamamoto, Y.; Suzuki, T.; Signorell, R. Genuine binding energy of the hydrated electron. Sci. Adv. 2017, 3, No. e1603224.

(12) Ottosson, N.; Faubel, M.; Bradforth, S. E.; Jungwirth, P.; Winter, B. Photoelectron spectroscopy of liquid water and aqueous solution: Electron effective attenuation lengths and emission-angle anisotropy. J. Electron Spectrosc. Relat. Phenom. 2010, 177, 60-70.

(13) Signorell, R. Electron scattering in liquid water and amorphous ice: A striking resemblance. Phys. Rev. Lett. 2020, 124, 205501.

(14) Bardeen, C. J. The structure and dynamics of molecular excitons. Annu. Rev. Phys. Chem. 2014, 65, 127-148.

(15) Pieniazek, P. A.; Sundstrom, E. J.; Bradforth, S. E.; Krylov, A. I. The degree of initial hole localization/delocalization in ionized water clusters. J. Phys. Chem. A 2009, 113, 4423-4429. 
(16) Barth, S.; Ončák, M.; Ulrich, V.; Mucke, M.; Lischke, T.; Slavíček, P.; Hergenhahn, U. Valence ionization of water clusters: From isolated molecules to bulk. J. Phys. Chem. A 2009, 113, 13519- 13527.

(17) Cabral do Couto, P.; Chipman, D. M. Insights into the ultraviolet spectrum of liquid water from model calculations. J. Chem. Phys. 2010, 132, 244307.

(18) Gaiduk, A. P.; Pham, T. A.; Govoni, M.; Paesani, F.; Galli, G. Electron affinity of liquid water. Nat. Commun. 2018, 9, 247.

(19) Thürmer, S.; Seidel, R.; Faubel, M.; Eberhardt, W.; Hemminger, J. C.; Bradforth, S. E.; Winter, B. Photoelectron angular distributions from liquid water: Effects of electron scattering. Phys. Rev. Lett. 2013, 111, 173005.

(20) Zhang, C.; Andersson, T.; Förstel, M.; Mucke, M.; Arion, T.; Tchaplyguine, M.; Björneholm, O.; Hergenhahn, U. The photoelectron angular distribution of water clusters. J. Chem. Phys. 2013, 138, 234306.

(21) Hartweg, S.; Yoder, B. L.; Garcia, G. A.; Nahon, L.; Signorell, R. Size-resolved photoelectron anisotropy of gas phase water clusters and predictions for liquid water. Phys. Rev. Lett. 2017, 118, 103402.

(22) Nishitani, J.; West, C. W.; Suzuki, T. Angle-resolved photoemission spectroscopy of liquid water at 29.5 eV. Struct. Dyn. 2017, 4, No. 044014.

(23) Gartmann, T. E.; Hartweg, S.; Ban, L.; Chasovskikh, E.; Yoder, B. L.; Signorell, R. Electron scattering in large water clusters from photoelectron imaging with high harmonic radiation. Phys. Chem. Chem. Phys. 2018, 20, 16364-16371.

(24) Jablonski, A.; Powell, C. Relationships between electron inelastic mean free paths, effective attenuation lengths, and mean escape depths. J. Electron Spectrosc. Relat. Phenom. 1999, 100, 137- 160 .

(25) Michaud, M.; Wen, A.; Sanche, L. Cross sections for lowenergy (1-100 eV) electron elastic and inelastic scattering in amorphous ice. Radiat. Res. 2003, 159, 3-22.

(26) Schild, A.; Peper, M.; Perry, C.; Rattenbacher, D.; Wörner, H. J. Alternative approach for the determination of mean free paths of electron scattering in liquid water based on experimental data. J. Phys. Chem. Lett. 2020, 11, 1128-1134.

(27) Stener, M.; Fronzoni, G.; Toffoli, D.; Decleva, P. Time dependent density functional photoionization of $\mathrm{CH} 4, \mathrm{NH} 3, \mathrm{H} 2 \mathrm{O}$ and HF. Chem. Phys. 2002, 282, 337-351.

(28) Ahmed, M.; Kostko, O. From atoms to aerosols: Probing clusters and nanoparticles with synchrotron based mass spectrometry and X-ray spectroscopy. Phys. Chem. Chem. Phys. 2020, $22,2713-2737$.

(29) Kostko, O.; Jacobs, M. I.; Xu, B.; Wilson, K. R.; Ahmed, M. Velocity map imaging of inelastic and elastic low energy electron scattering in organic nanoparticles. J. Chem. Phys. 2019, 151, 184702. 
(30) Truesdale, C. M.; Southworth, S.; Kobrin, P. H.; Lindle, D. W.; Thornton, G.; Shirley, D. A. Photoelectron angular distributions of H2O. J. Chem. Phys. 1982, 76, 860.

(31) Banna, M. S.; McQuaide, B. H.; Malutzki, R.; Schmidt, V. The photoelectron spectrum of water in the 30 to $140 \mathrm{eV}$ photon energy range. J. Chem. Phys. 1986, 84, 4739.

(32) Faubel, M.; Siefermann, K. R.; Liu, Y.; Abel, B. Ultrafast soft Xray photoelectron spectroscopy at liquid water microjets. Acc. Chem. Res. 2012, 45, 120-130.

(33) Gozem, S.; Gunina, A. O.; Ichino, T.; Osborn, D. L.; Stanton, J. F.; Krylov, A. I. Photoelectron wave function in photoionization: Plane wave or Coulomb wave? J. Phys. Chem. Lett. 2015, 6, $4532-4540$.

(34) Gozem, S.; Krylov, A. I. ezDyson User's Manual. ezDyson, 2015; http://iopenshell.usc.edu/downloads/ (accessed April 2018).

(35) Martini, L.; Boll, D. I. R.; Fojón, O. A. Interferences in the photoionization of water molecules. J. Phys. B: At., Mol. Opt. Phys. 2019, 52, 105204.

(36) Belkić, D.; Gayet, R.; Salin, A. Electron capture in high-energy ion-atom collisions. Phys. Rep. 1979, 56, 279.

(37) Ciappina, M. F.; Fojón, O. A.; Rivarola, R. D. Coherent electron emission from simple molecules by impact of energetic charged particles. J. Phys. B: At., Mol. Opt. Phys. 2014, 47, No. 042001.

(38) Baltenkov, A. S.; Dolmatov, V. K.; Manson, S. T. Multicentered theory of molecular photoionization. Surf. Rev. Lett. 2002, 9, 1143- 1148.

(39) Baltenkov, A. S.; Manson, S. T.; Msezane, A. Z. Elastic scattering of particle by a system of non-overlapping spherically symmetric potentials. J. Phys. B: At., Mol. Opt. Phys. 2007, 40, 769777.

(40) Mabbs, R.; Pichugin, K.; Sanov, A. Dynamic molecular interferometer: Probe of inversion symmetry in 12 - photodissociation. J. Chem. Phys. 2005, 123, No. 054329.

(41) Winter, B.; Weber, R.; Widdra, W.; Dittmar, M.; Faubel, M.; Hertel, I. V. Full valence band photoemission from liquid water using EUV synchrotron radiation. J. Phys. Chem. A 2004, 108, 2625-2631. 